\title{
Integrated Sensing Systems for Monitoring Interrelated Physiological Parameters in Young and Aged Adults: A Pilot Study
}

\author{
Mark Sprowls ${ }^{1,2 * *}$, Michael Serhan ${ }^{1,2 * *}$, En-Fan Chou ${ }^{3}$, Lancy Lin $^{3}$, Christopher Frames ${ }^{6}$, Ivan Kucherenko ${ }^{7,8}$, Keyvan $^{6}$ \\ Mollaeian $^{7}$, Yang Li ${ }^{10}$, Varun Jammula ${ }^{4,5}$,Dhenugen Logeswaran ${ }^{10}$, Michelle Khine ${ }^{3}$, Yezhou Yang ${ }^{4,5}$, Thurmon Lockhart ${ }^{6}$, \\ Jonathan Claussen $^{7,16}$, Liang Dong ${ }^{9}$, Julian J.-L. Chen ${ }^{10}$, Juan Ren ${ }^{7}$, Carmen Gomes ${ }^{7}$, Daejin Kim ${ }^{15}$, Teresa Wu ${ }^{13,14}$, Jennifer \\ Margrett ${ }^{12^{*}}$, Balaji Narasimhan ${ }^{11^{*}}$, Erica Forzani ${ }^{1,2^{*}}$ \\ ${ }^{1}$ School for Engineering of Matter, Transport, and Energy, Arizona State University, Tempe, AZ, USA \\ ${ }^{2}$ Center for Bioelectronics and Biosensors, Biodesign Institute, Arizona State University, Tempe, AZ, USA \\ mark.sprowls@asu.edu; mserhan@asu.edu; eforzani@asu.edu \\ ${ }^{3}$ Department of Biomedical Engineering, University of California Irvine, Irvine, CA 92697, USA \\ enfanc@uci.edu; lancyl@uci.edu; mkhine@uci.edu
}

${ }^{4}$ School of Computing, Informatics, and Decision Systems Engineering, Arizona State University, Tempe, AZ, USA

${ }^{5}$ Active Perception Group, CIDSE, Arizona State University, Tempe, AZ, USA

vjammula@asu.edu; yz.yang@asu.edu

${ }^{6}$ School of Biological and Health Systems Engineering, Arizona State University, Tempe, AZ, USA

cframes@asu.edu;Thurmon.Lockhart@asu.edu

${ }^{7}$ Department of Mechanical Engineering, Iowa State University, Ames, IA 50011, USA

${ }^{8}$ Department of Biomolecular Electronics, Institute of Molecular Biology and Genetics of the National Academy of Sciences of Ukraine, Zabolotnogo Street 150, Kyiv, Ukraine

ivan@iastate.edu; keyvanm@iastate.edu; jcclauss@iastate.edu; juanren@iastate.edu; carmen@iastate.edu

9Department of Electrical and Computer Engineering, Iowa State University, Ames, IA 50011, USA

ldong@iastate.edu

10School of Molecular Sciences, Arizona State University, Tempe, AZ, USA

yanglena.li@gmail.com; dlogeswa@asu.edu; Julian.Chen@asu.edu

${ }^{11}$ Department of Chemical and Biological Engineering, Iowa State University, Ames, Iowa 50011

nbalaji@iastate.edu

${ }^{12}$ Department of Human Development and Family Studies, Iowa State University, Ames, IA 50011, USA

margrett@iastate.edu

${ }^{13}$ School of Computing, Informatics, Decision Systems Engineering, Arizona State University, Tempe, AZ, USA

${ }^{14}$ ASU-Mayo Center for Innovative Imaging, Arizona State University, Tempe, AZ, USA

Teresa.Wu@asu.edu

${ }^{15}$ Department of Interior Design, Iowa State University, Ames, IA 50011, USA

daejink@iastate.edu

\footnotetext{
Mark Sprowls et al. This is an open-access article distributed under the terms of the Creative Commons Attribution 3.0 United States License, which permits unrestricted use, distribution, and reproduction in any medium, provided the original author and source are credited.

https://doi.org/10.36001/IJPHM.2021.v12i4.2914
}

International Journal of Prognostics and Health Management, ISSN 2153-2648, 2021 


\section{Abstract}

Acute injury to aged individuals represents a significant challenge to the global healthcare community as these injuries are frequently treated in a reactive method due to the infeasibility of frequent visits to the hospital for biometric monitoring. However, there is potential to prevent a large number of these cases through passive, at-home monitoring of multiple physiological parameters related to various causes that are common to aged adults in general. This research strives to implement wearable devices, ambient "smart home" devices, and minimally invasive blood and urine analysis to test the feasibility of implementation of a multitude of research-level (i.e. not yet clinically validated) methods simultaneously in a "smart system". The system comprises measures of balance, breathing, heart rate, metabolic rate, joint flexibility, hydration, and physical performance functions in addition to lab testing related to biological aging and mechanical cell strength. A proof-ofconcept test is illustrated for two adult males of different ages: a 22-year-old and a 73-year-old matched in body mass index (BMI). The integrated system is test in this work, a pilot study, demonstrating functionality and age-related clinical relevance. The two subjects had physiological measurements taken in several settings during the pilot study: seated, biking, and lying down. Balance measurements indicated changes in sway area of $45.45 \%$ and $25.44 \%$, respectively for before/after biking. The 22-year-old and the 73-year-old saw heart rate variabilities of 0.11 and 0.02 seconds at resting conditions, and metabolic rate changes of $277.38 \%$ and $222.23 \%$, respectively, in comparison between the biking and seated conditions. A smart camera was used to assess biking speed and the 22- and 73-year-old subjects biked at $60 \mathrm{rpm}$ and $28.5 \mathrm{rpm}$, respectively. The 22 -year-old subject saw a 7 times greater electrical resistance change using a joint flexibility sensor inside of their index finger in comparison with the 73-year-old male. The 22 and 73-year-old males saw respective $28 \%$ and $48 \%$ increases in their urine ammonium concentration before/after the experiment. The average lengths of the telomere DNA from the two subjects were measured to be $12.1 \mathrm{~kb}$ (22-year-old) and $6.9 \mathrm{~kb}$ (73-yearold), consistent with their biological ages. The study probed feasibility of 1) multi-metric assessment under free living conditions, and 2) tracking of the various metrics over time.

\section{INTRODUCTION}

By 2030, the number of U.S. older adults is projected to increase from 35 million to 72 million (from $12 \%$ to $20 \%$ of the population).

By 2035, there will be more Americans over 65 than under 18 (U.S. Census Bureau "Older People Projected to Outnumber Children for First Time in U.S. History" 2018). The majority of older adults prefer to remain in their home for as long as possible preventing a need to relocate to a supportive living setting (e.g., assisted living) as long as their safety, independence, and comfort are not compromised (Binette \& Vasold, 2018). Given that aging adults are generally at higher risk for a variety of safety and health issues (Perdue, Watts Dd Fau - Kaufmann, Kaufmann Cr Fau - Trask, \& Trask, 1998), the primary challenge for successful, independent "aging in place" is an understanding of risk factors in the home that can be minimized to promote safety, well-being, and everyday functioning. In this context, mobility and physiological functioning represent intertwined foundational functions that most impact older adults' daily lives. Mobility impairment increases fall risk, loss in functional capacity, isolation, and mortality.(Boulgarides, McGinty, Willet, \& Barnes, 2003; Daubney \& Culham, 1999; Musich, Wang, Ruiz, Hawkins, \& Wicker, 2018; ShumwayCook, Brauer, \& Woollacott, 2000). Even though human physiologies are characterized by multiple, interacting physiological systems, current medical technologies are largely lacking in cross-modal ability. Multi parametric sensor systems are now a true possibility with the recent technological advancements in IoT technology, cloud computing, and machine learning algorithms for development of predictive disease models.

At the fundamental level, the scientific community must address the challenge of acquiring high-fidelity measurements of biosignals that generally decay in amplitude as individuals age. Over time biosignals often become more difficult to detect, noisy, and complex in our target population (Charlton et al., 2017). For example, it is well established that extracting accurate breath rate from photoplethysmogram signals in older populations (> 70 years of age) is more difficult than with younger groups (Charlton et al., 2017). Similarly, sweat analysis in older populations is more challenging because of reduced water storage due to reduced urge to drink fluids among older individuals as well as physiological changes in water storage (Popkin, D'Anci Ke Fau - Rosenberg, \& Rosenberg, 2010). Monitoring key physiological and biomechanical parameters with sufficient signal-to-noise ratio as well as with the unique capability of assessing physiologically-related data using multiple, integrated sensors provides us with the ability to obtain a truly comprehensive personal health assessment without eschewing valuable physiological data that is often lost when only a single medical device is used. Key physiological parameters employed in monitoring the health of aging adults include longitudinal cardiovascular, respiratory, postural stability, hydration, hormone balance, metabolic rate, mobility, and motor function indicators. Dehydration is specifically a significant challenge for aging adults as it is often asymptomatic and approximately 40-60\% are chronically dehydrated (Bennett, Thomas, \& Riegel, 2004; Picetti et al., 2017). In some age-associated chronic diseases, the level of dehydration may become severe enough to lower blood pressure and result in falls (Picetti et al., 2017) as well as increased mortality rates among hospitalized patients (Lavizzo-Mourey, 1987). Additionally, dehydration is a 
major cause of general fatigue (Riebl \& Davy, 2013), that may also be interrelated to metabolic rate (Woods, GarvicanLewis, Lundy, Rice, \& Thompson, 2017).

In an effort to overcome the above-mentioned challenges, our team has integrated, multimodal physiological functioning measurements that can capture multiple sensitive responses with precision, speed, reliability, and security that cannot be achieved using a single modality sensor. Specifically, we introduce a single integrated sensing system, which is comprised of wearables and ambient sensors for assessment of an individual's physiological parameters in addition to environmental parameters. The system includes a diverse source of sensing transduction mechanisms as summarized in Table 1 and it was tested in a simulated, free-living space. The integrated system is primarily envisioned NOT as a tool for clinical diagnoses. On the contrary, the integrated system provides a tool for daily monitoring, early diagnosis of physiological data that is outside the expected healthy baseline and stimulus for the user to follow up with a clinician for further diagnosis. These types of multiparametric regressions for prediction of the occurrence of various diseases has not yet been completed to date, primarily due to the lack of an integrated sensing system for this purpose. As such, the proposed work strives to provide pilot data to illustrate the capability of such a system.

The integrated system was tested in this work, a pilot study, demonstrating functionality and age-related clinical relevance. The study probed feasibility of: 1) multi-metric assessment under conditions simulating free living conditions, and 2) tracking of various metrics over time. Additionally, the study enabled an initial quantitative understanding of health baseline for various biometrics as it relates to subject age for males with the given BMI characteristics. In the future, further studies on a broad population of age, sex, and ethnicities will warrantee to provide more conclusive data on baseline biometrics for healthy subjects.

Many of the devices and techniques used in this study are novel and are already undergoing study for accuracy among various other metrics common to medical devices (see each experimental section for more detail). For that reason, the goal of this study was not to validate each device with any type of reference instrument. Rather, the true value of this study is that is shows how multiple, integrated devices that are approaching clinical application can be simultaneously utilized to paint a broad picture of a person's health in a noninvasive manner. Many technical challenges require innovative engineering approaches to provide an accurate analytical result for the proposed autonomous integrated sensing system for health monitoring of older adults (e.g. discrimination of various individuals within in the environment), and, these will undoubtedly be the focus of future works.

\section{EXPERIMENTAL SECTION}

\subsection{Human subjects}

The integrative sensor system described in Table 1 was assembled in a single location to simultaneously collect raw sensor data in a time-stamped fashion. After data collection, the data was synchronized for further analysis. Study participants of different ages (ranging from 22-75-year-old) were recruited via ASU's IRB number STUDY00006547 and provided with sensors (shown in Fig 1A) for simultaneous assessment of physical and chemical parameters while performing a sequence of predetermined tasks under three conditions (shown in Fig 1B): 1. Resting (sitting in a chair), 2. Activity (fixed biking), 3. Supine (lying down). In addition, participants' biological samples (urine/blood) were collected before and after the tests and analyzed for osmolarity and relevant biomarkers indicative of cardiac function. One subject was aged 73 years old, $74.84 \mathrm{~kg}, 167.64 \mathrm{~cm}$, and a BMI of 26.6. The other subject was aged 22 years, $81.65 \mathrm{~kg}$, $177.8 \mathrm{~cm}$, and had a BMI of 25.8. Neither subject had any serious health concerns at the time of the assessment, other than the older male who suffered from mild arthritis.

\subsection{Sensors of the integrated sensing system}

The research team integrated a set of different wearables, offbody sensors embedded in the room environments, and blood based complementary sensors. All of these sensors are briefly described in Table 1, but enumerated in more detail below.

\subsubsection{Balance Sensor (Wearable)}

The balance sensor is comprised of an iPhone-based 3-axis accelerometer, acting as inertial measurement unit (IMU) that provides real-time and continuous data acquisition. The iPhone is equipped with an app called Lockhart Monitor developed by the co-authors (Fig. 2).

All measurements were performed in quiet standing with standardized feet placement, looking in the forward direction. Participants were tasked with standing in two visual conditions: eyes-open (EO) and eyes-closed (EC). Measurements were taken on both subjects prior to the biking assessment and compared to the same set of measurements performed immediately following the biking activity. Each measurement lasted for 60 seconds and was repeated twice. The sampling rate was $100 \mathrm{~Hz}$. A rest of two minutes was afforded between each measurement. For the analysis, the center of pressure (COP) trajectory was separated into its mediolateral (ML) and anteroposterior (AP) components. The recorded COP signals were filtered using a third-order low-pass Butterworth filter, with a cutoff frequency of $5 \mathrm{~Hz}$ to eliminate measurement noise. Given the limited data length, measurements began a few seconds after the informed start of the trial and ended a few seconds before the informed termination of the trial. All analysis was performed using 
custom Matlab routines (The Mathworks, Version 2018a) as well as the Lockhart Monitor App. More details can be found in additional publications by the co-authors: (Frames et al., 2018); (Lockhart, Soangra, \& Frames, 2014).

\subsubsection{SmartPad Sensor (Environmental)}

This system provides metabolic rate [kcal/day] assessment through measurements of $\mathrm{CO}_{2}$ concentration within the room while the subject is present. Though measurements of ambient $\mathrm{CO}_{2}$ production rate $\left(k_{\text {gen }}[\mathrm{ppm} / \mathrm{hour}]\right), \mathrm{CO}_{2}$ production rate in volumetric terms $\left(\mathrm{VCO}_{2}[\mathrm{~mL} / \mathrm{min}]\right)$ can be estimated, and finally this term can then be used for metabolic rate [kcal/day] estimates. More details on this system have been described previously by the authors (Ruiz et al., 2018). The system is fully environmentally based (i.e., no wearables) and uses the seat pad with integrated strain sensors optionally to provide additional postural data if the subject is present.

Table 1. Components of Integrated Sensing System

\begin{tabular}{|c|c|c|}
\hline Sensor Type & Sensor & Measured Parameters \\
\hline Wearable & Balance Sensor & $\begin{array}{l}\text { Sway Path }(\mathrm{cm}) \\
\text { Sway Velocity }(\mathrm{cm} / \mathrm{s}) \\
\text { Sway Area }\left(\mathrm{cm}^{2}\right)\end{array}$ \\
\hline Wearable & Capacitive Pressure Sensor & $\begin{array}{l}\text { Systolic Blood Pressure (mm Hg) } \\
\text { Diastolic Blood Pressure (mm Hg) }\end{array}$ \\
\hline Wearable & $\begin{array}{l}\text { Electrocardiography (Bioelectrical Signal } \\
\text { Sensor) }\end{array}$ & Heart Rate Variability (s) \\
\hline Wearable & Strain-based Respiratory sensor & End Tidal Volume (mL) \\
\hline Wearable & Joint Flexibility Sensor & Resistance $(\mathrm{k} \Omega)$ \\
\hline Environmental & SmartPad System (on a chair) & $\begin{array}{l}\text { Metabolic Rate (kcal/day) } \\
\text { Temperature }\left({ }^{\circ} \mathrm{C}\right) \\
\text { Relative Humidity }(\%)\end{array}$ \\
\hline Environmental & Smart Camera (on a table) & Video (body joint position) \\
\hline Environmental & Smart Toilet & Ammonium and Potassium Concentration (mM) \\
\hline In Vitro (Blood) & Telomere Length Test & Telomere Length $(\mathrm{kb})$ \\
\hline In Vitro (Tissue) & Cellular Mechanical Strength Test & Young's Modulus (Pa) \\
\hline
\end{tabular}

A

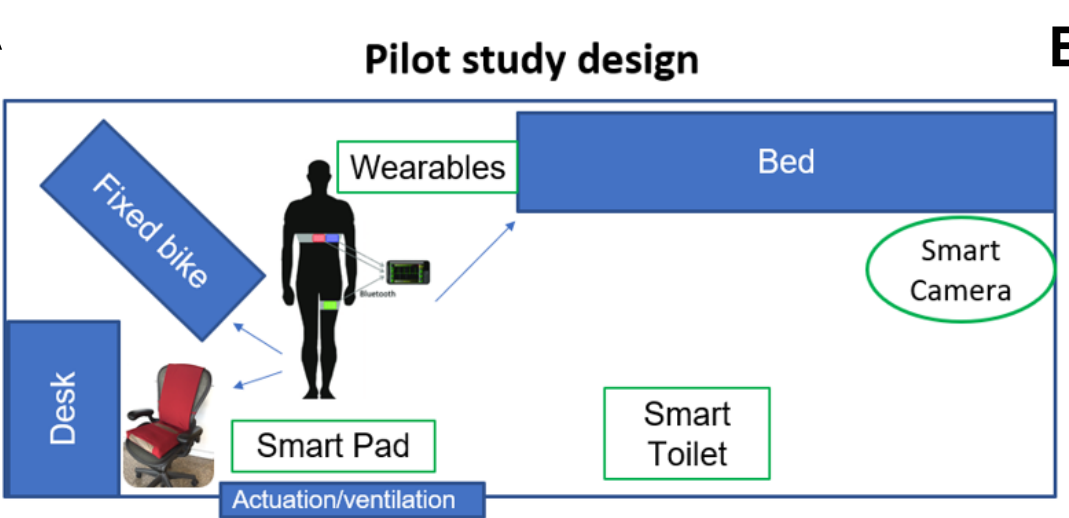

B Sequence of Events:

1. Sitting (60 minutes)
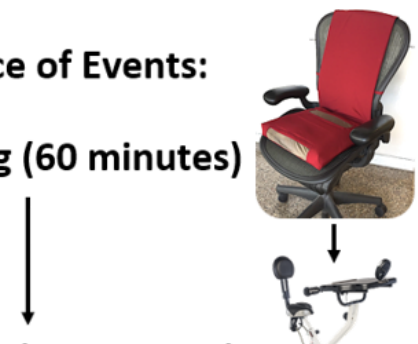

2. Biking (10 minutes)

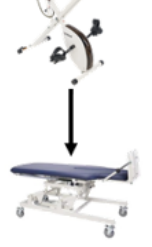

Fig. 1A. Schematic representation of integrated sensing system and pilot study design. Fig 1B. Sequence of events followed by subject during experiment. 
During the metabolic assessment, the system controls the ventilation through a fan system within the room, which turns ON/OFF after fixed intervals of time. The fan system consists of an inlet and outlet fan that cycles fresh air into the room when the system is turned on (as observed in the data shown in Fig 7A in the form of dropping $\mathrm{CO}_{2}$ concentrations following data assessments) to reduce any effects of cognitive poisoning due to high $\mathrm{CO}_{2}$ concentration (or other bio effluents). The fan system is turned OFF prior to each metabolic assessment to allow $\mathrm{CO}_{2}$ levels within the room to increase, since increasing $\mathrm{CO}_{2}$ concentration with time is the signal that allows the SmartPad system to assess metabolic rate. Other than the fan system, the room was specially sealed to ensure no effect of other uncontrolled ventilation variables. This sealing includes a specially made door to prevent air leakage. All data were assessed using OriginPro 2017 $\left(\right.$ OriginLab $\left.^{\mathrm{TM}}\right)$.

Still in development, this passive sensing system is eventually envisioned to see deployment in any person's home office or bedroom (or alternatively, a clinician's attending room). In the home environment, the system would require integration in the house's HVAC system. Future work on the SmartPad will largely be focused on development of AI algorithms for necessary corrections of various environmental occurrences, including periodic AC actuation within an individual's home. In the final target application, the SmartPad itself will not consist of an actuation/fan system.

\subsubsection{Ribcage/Abdomen Respiratory Sensors, Electrocardiography Sensor \& Wrist Blood Pressure Sensor (Wearables)}

Wearable health sensors with skin-like form factors have been developed to continuously monitor physiological vitals such as respiration and blood pressure (Chu et al., 2019; Kim et al., 2019). The respiration monitor consists of a system of piezoresistive strain sensors attached to the torso that generates a signal from detected diaphragm and abdomen expansion and contraction. We previously demonstrated that this system can be used to calculate respiration rate and volume (Chu et al., 2019). The blood pressure monitor is a skin-mounted, capacitive-based pressure sensor that picks up superficial arterial pulsatile waveforms. The calibrated waveforms are used to calculate continuous heart rate, blood pressure, and subsidiary parameters.

Respiration rate is commonly expressed as the number of breaths per minute and is inversely proportional to the time difference between consecutive peaks of the sinusoidal signal. Tidal volume is the amount of air displaced during exhalation and inhalation and is calculated by integrating the respiration airflow with respect to time. Heart rate is expressed as the number of heart beats per minute and is calculated from the continuous pulsatile waveform or electrocardiogram (ECG). Heart rate variability (HRV) is the time variance of consecutive heartbeats. Pulse transit time (PTT) measures the pulse wave traversal time from one arterial site to another.

Respiration, blood pressure, heart rate, and ECG were simultaneously monitored during various activities. Respiration was monitored using two strain sensors and a spirometer (BIOPAC, Inc.). The strain sensors were adhered onto the ribcage and abdomen regions using skin-safe adhesives. The spirometer was fitted with a disposable breathing filter and used in conjunction with a nose clip. The spirometer only serves to calibrate the respiration sensors and is not included in the final assessment of either subject's health. Blood pressure was monitored using a capacitive sensor mounted over the radial artery and a brachial arm cuff (OMRON Corp.). The capacitive sensor was secured in place using a wrist strap and the wrist was immobilized in a flexed position using an arterial line splint; an accelerometer and gyroscope (MC10, Inc.) was placed near the sensor to detect motion artifacts. The brachial arm cuff was placed on the opposite arm. ECG with conventional $\mathrm{Ag} / \mathrm{AgCl}$ electrodes (BIOPAC, Inc.) was monitored in a Lead I configuration with the positive and negative leads placed over the clavicles and the ground lead placed over the ribcage opposite the strain sensor. Figure 3A summarizes the locations where sensors were placed during the measurements.

Subjects were tested in three positions for various activities: relaxed sitting for the cognitive stress test, upright exercise biking for the physical stress test, and supine on a $45 \mathrm{deg}$ recline for post-stress recovery. Each position was recorded for approximately 10 minutes. At the beginning of the relaxed sitting position, subjects were instructed to breathe into the spirometer at slow, normal, and fast paces for 1 minute each. This data was used to calibrate the strain sensors to the spirometer. After this period, the spirometer and nose clip were removed for the rest of the testing period.

Data analysis was performed using MATLAB (MathWorks). Three blood pressure measurements were taken by the brachial arm cuff before the beginning of the testing. The averaged systolic and diastolic values from three measurements were used as calibration for the first waveform.

\subsubsection{Smart Camera (Environmental)}

We used a stereo camera (https://www.stereolabs.com/zed) set up to collect data. The stereo camera setup comes with two monocular cameras arranged parallel to each other. The baseline distance between the monocular cameras is $120 \mathrm{~mm}$. The camera collects video data with a resolution of $1920 \times 1080$ at 20 frames per second (fps). Along with the camera, we use Human Mesh Recovery (Kanazawa, Black, Jacobs, \& Malik, 2018) for reconstructing a full 3D mesh of a human body from a single RGB image. For each frame from the video, we obtain a human mesh according to SMPL model (Loper, Mahmood, Romero, Pons-Moll, \& Black, 
2015). The obtained 3D joint angles consist of roll, pitch, and yaw values for every joint. In this study, we recorded the pitch of the knee joint at every frame and obtained the frequency of biking. This sensor is not attached to the subject but rather placed on a table in the environment to capture the subject motion. The camera is set up in such a way that it can capture the part of the human body performing an activity. For example, when the subjects are performing biking exercises, the camera is positioned in such a way that it can observe the lower body of the subject. The camera was positioned in a single location on a wall during the entire experiment and did not require repositioning. From that location on the wall, the camera could capture nearly a full view of all objects within the room and its boundaries.

\subsubsection{Joint Flexibility Sensors (Wearable)}

Information related to sensor fabrication can be found in (Oren, Ceylan, Schnable, \& Dong, 2017). This graphene sensor's electrical resistance changes significantly upon deflection. Results are shown for the young and aged subject during the study, with the sensor placed inside the finger of the subject. Each subject was instructed to bend their index finger maximally. The finger flexibility assessment was performed immediately prior to any activity described in 2.1 (before the resting assessment).

\subsubsection{Smart Toilet Sensors (Environmental)}

Graphene-based, solid-state ion selective sensors were created via laser induction of polyimide following our previously delineated protocols. Two parallel electrodes were lased on the polyimide with a UV laser. Multiple replicates of the parallel electrodes to obtain statistically significant results and to create distinct ion sensors, one for potassium $\left(\mathrm{K}^{+}\right)$ion sensing and one for ammonium $\left(\mathrm{NH}_{4}^{+}\right)$. For example, one replicate was functionalized with a drop cast of polyvinyl chloride (PVC)-based $\mathrm{K}^{+}$selective membrane on one electrode and with a screen-printed $\mathrm{Ag} / \mathrm{AgCl}$ paste on the parallel electrode to create a solid-state ion selective sensor for $\mathrm{K}^{+}$with a corresponding $\mathrm{Ag} / \mathrm{AgCl}$ reference electrode. In a similar fashion another replicate was functionalized with $\mathrm{NH}_{4}{ }^{+}$-based ion selective sensor and an $\mathrm{Ag} / \mathrm{AgCl}$ reference electrode. It is important to note these sensors are flexible and multiple bending cycles have been performed to demonstrate that the electrodes show negligible loss in electrical conductivity after bending. Thus, these electrodes could be placed on the curvilinear surface of a toilet for example. The sensors are operated with an electrochemical potentiometric sensing modality and hence quantify the concentrations of both $\mathrm{K}^{+}$and $\mathrm{NH}_{4}{ }^{+}$in solutions by being wired to a portable potentiostat chip that is powered by a battery. The Smart Toilet measurements were taken by simply collecting urine samples from subjects and analyzing in a laboratory setting. The measurement is currently in the form of a laboratory test, but, future work will strive to integrate this measurement in a toilet to perform effortless measurements on subject urine.
Smart toilet measurements were taken immediately before and after each subject's biking assessment.

\subsection{In-vitro methods}

\subsubsection{Telomere Length}

Genomic DNA was isolated from peripheral blood mononuclear cells (PBMC) and used for average telomere length measurement using the terminal restriction fragment (TRF) analysis (Mender \& Shay, 2015). Briefly, the PBMC were isolated from $7 \mathrm{~mL}$ of whole blood using the LeucoSep Tube following manufacturer's instructions. Genomic DNA was extracted from $4 \times 10^{6}$ of PBMC cells with Wizard genomic DNA purification kit (Promega). Five $\mu \mathrm{g}$ of genomic DNA was digested with 10 units of Alu-I and Rsa-I each at $37^{\circ} \mathrm{C}$ for $3 \mathrm{~h}$. The digested genomic DNA samples were resolved on a $0.8 \%$ agarose gel, followed by in-gel hybridization using telomere probe (TTAGGG) ${ }_{3}$ (Mender \& Shay, 2015). The gel was then scanned using Typhoon IP (GE Healthcare) scanner. The scanned gel image was analyzed using the TeloTool program (Gohring, Fulcher N Fau - Jacak, Jacak J Fau - Riha, \& Riha, 2014) to determine the mean telomere lengths. This assay could be automated so that the method could be implemented as a home-based instrument to perform the analysis from finger picked whole blood samples. Not only is mean telomerase length expected to increase with age (which is interesting from a research perspective) but may have clinical significance for a variety of age-related disease such as atherosclerosis (Benetos et al., 2004), myocardial infarction (Brouilette, Singh, Thompson, Goodall, \& Samani, 2003), and Alzheimer's disease (Panossian et al., 2003).

\subsubsection{Atomic force microscopy (AFM) stiffness measurement of fruit fly heart tissue}

Since the heart is a vital part of any living organism and its mechanical properties certainly affect its performance, this study also explored the feasibility of using a new research diagnostic tool for the stiffness of different region of the heart tissue. Several fruit fly hearts were studied to determine if there was a significant difference between the mechanical properties of aged and young fruit fly hearts. The findings from this study provided some insight onto hypothesized differences in mechanical properties of human hearts and these properties' dependence on age. We are also exploring the mechanical properties of white blood cells with the idea that this in-vitro method could be also automated and implemented as a test in a home-based instrument to perform the analysis from finger picked whole blood samples. AFM measurements were performed in phosphate-buffered saline (PBS) at room temperature using Bruker BioScope Resolve AFM system (Santa Barbara, CA). Glass sphere bead probe (Novascan, IA, USA) with the radius of $2.5 \mu \mathrm{m}$ was used. A cantilever spring constant of $0.03 \mathrm{~N} / \mathrm{m}$ was acquired using the thermal tune approach. To minimize the effect of the fruit fly 
heart thickness and substrate, the target indentation depth was selected as $1300 \mathrm{~nm}$, which was less than a quarter of the heart height at $0.5 \pm 0.1 \mathrm{~mm}$ (Chan, Hu, Johnson, Suo, \& Stafford, 2012; Dimitriadis, Horkay, Maresca, Kachar, \& Chadwick, 2002). The measurements were performed on four segments of the heart (i.e., A1-A4) on at least sixteen different flies for each age group of six days, and seven weeks (i.e., young and aged flies, respectively). The stiffness (i.e., Young's modulus) was quantified using the approach reported in (Mollaeian et al., 2018). Although usage of AFM for measure of cellular stiffness of heart tissue is admittedly far from an at home system for measurement physiological metrics, the authors envision the basic scientific principles of such a technique as a real possibility for at home monitoring instrument. Cellular stiffness has been previously shown in red blood cells (RBC) (Barns et al., 2017). A cellular stiffness test from RBC, which can be obtained from a finger prick, using an AFM-based bench top instrument is a certainty possible future instrument. As such, the inclusion of these AFM measurements poses an interesting, yet admittedly far from physical realization, potential development in minimally invasive point of care technologies.

\section{RESULTS AND DISCUSSION}

\subsection{Balance Sensor}

Balance (cell phone IMU-based) sensors were applied to evaluate the balance of the subjects before and after the physical challenge of biking for $10 \mathrm{~min}$. A summary of the results from two subjects paired in Body Mass Index (BMI) and gender but of different ages ( 22 and 73 year-old) is shown in Table 2. Overall, both subjects had normal fall risk indexes (shown in final row of Table 2). However, a closer look at the sway area as shown in Figure 2, indicated that the postural stability was about twice lower in the aged adult than the younger adult $\left(11.4 \mathrm{~cm}^{2}\right.$ vs $\left.6.7 \mathrm{~cm}^{2}\right)$. The older adult neared the cut-off value of high risk for fall occurrence shown in previous works, but, did not exceed it $\left(14.3 \mathrm{~cm}^{2}\right.$ vs cutoff value: $>15.7$ - references for expected fall risk values shown in final row of table 2). All other (i.e., non-balance related) wearable sensors were used simultaneously by the subjects while performing different activities (see below), whereas the balance sensor was only used before/after the biking activity.
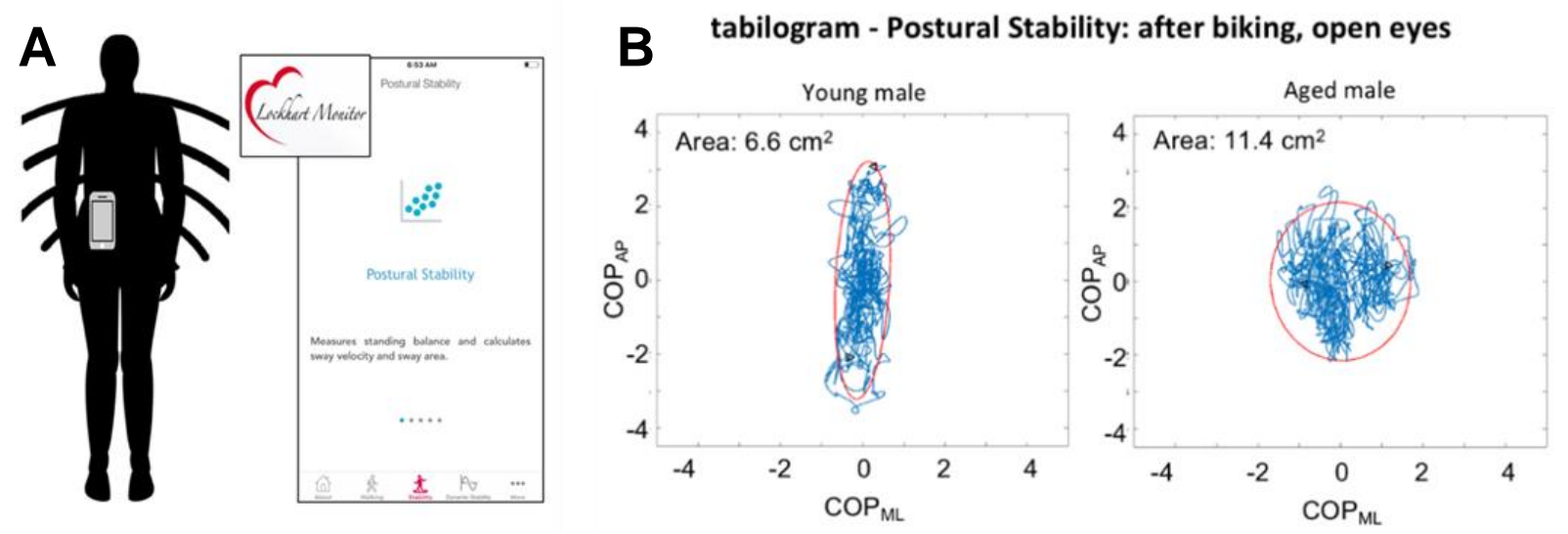

Figure 2A. Stabilogram was built by using the mobile App with IMU sensor. Figure 2B. Postural stability was evaluated after biking with open eyes for the subjects matched in BMI and sex

Table 2. Summary of balance measurements for two subjects with similar physiological characteristics (i.e., BMI)

\begin{tabular}{|l|l|l|l|l|}
\hline ID & Age & Sway Path $(\mathbf{c m})$ & Sway Velocity $(\mathbf{c m} / \mathbf{s})$ & Sway Area $\left.\mathbf{c m}^{2}\right)$ \\
\hline Young - pre & 22 & 181.4 & 3.0 & 6.6 \\
\hline Young - post & 22 & 217.7 & 3.6 & 9.6 \\
\hline Aged - pre & 73 & 255.1 & 4.2 & 11.4 \\
\hline Aged - post & 73 & 162.4 & 2.7 & 14.3 \\
\hline $\begin{array}{l}\text { Expected values for Non-fall risk } \\
\text { cases (Doheny et al., 2012; Gago } \\
\text { et al., 2014; Melzer, Benjuya, \& } \\
\text { Kaplanski, 2004) }\end{array}$ & $<355.5$ & $<7.15$ & $<15.7$ \\
\hline
\end{tabular}




\subsection{Ribcage/Abdomen Respiratory Sensor, Electrocardiogram Sensor and Wrist Blood Pressure Sensors}

Figure 3, 4 and 5 summarizes all the measurements from the wrist blood pressure, wearable breathing sensors, and heart rate sensors. Simultaneous data synchronization for all wearable sensors was demonstrated.
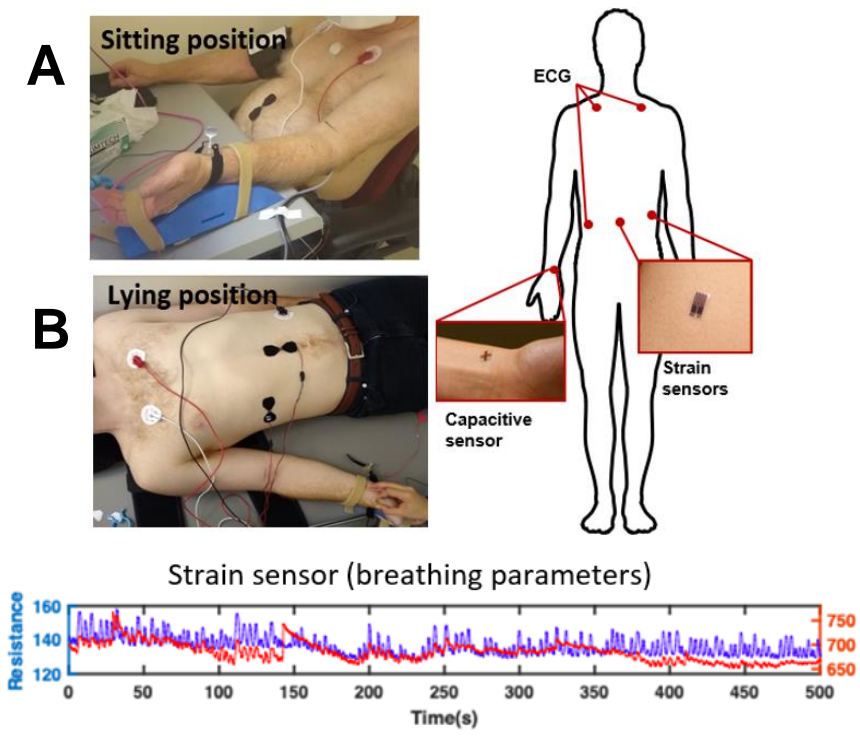

Figure 3. Physiological wearable sensors based on capacitance, strain, and a contact patch monitoring heart

function of electrocardiography. Strain sensor data for ribcage and abdomen, which is related to breathing rate and volume expired (ETV) in shown in bottom box.

The breathing sensors enabled the assessment of ETV for the subjects while seated and lying. The assessed values were compared with a another device (FDA approved) also designed to measure ETV (BreezingTM, (Mora, Bridgeman, Xian, Tao, \& Forzani, 2020)). The comparison rendered differences of $7.1 \%$ for the lying position in the younger individual and of $21.1 \%$ for the sitting position in the older individual. The results suggest some agreement between the two devices, although additional comparative measurements will be necessary in the future. ETV was not able to be measured during the biking period for either subject due to significant motion artifacts which could not be eliminated.

Figure 4 shows a snapshot of the results for the capacitance sensor, which was used for measurement of wrist blood pressure, for the young and aged individual respectively. Figure 4A shows representative waveform for a young male subject in comparison to an aged male subject (shown in Figure 4B). The capacitance sensor was previously calibrated with FDA cleared blood pressure meter $\left(\mathrm{Omrom}^{\mathrm{TM}}\right)$ and was able to provide real time blood pressure information.
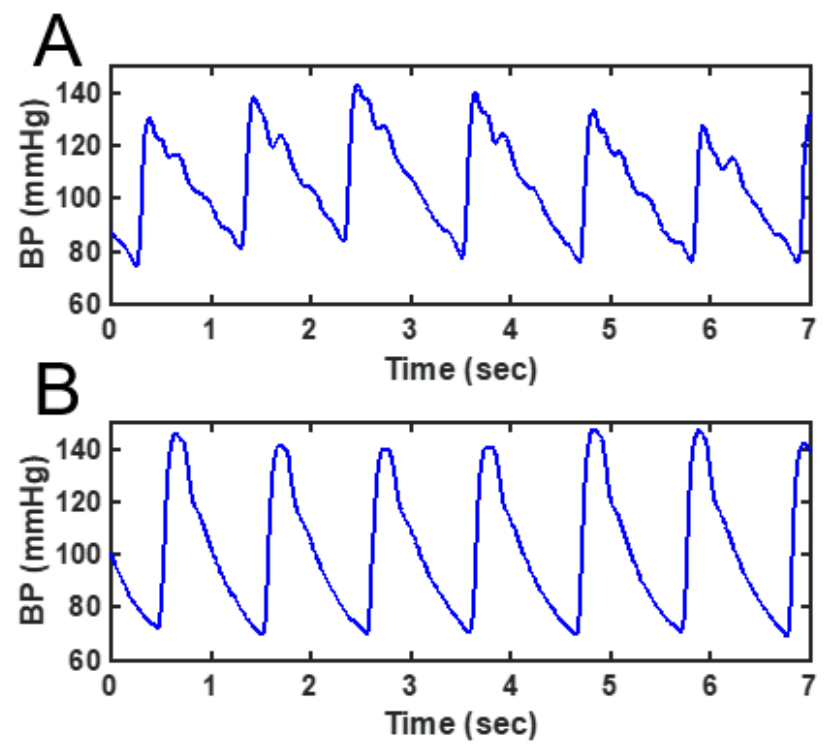

Figure 4. Physiological wearable sensors' signals and results for the young (Fig 4A) and the aged individuals (Fig 4B)

In addition, heart rate variability (HRV) was evaluated from R-R peak intervals (e.g., time in between beats) calculations from ECG data taken during the relaxed sitting portion of the testing. Poincaré plots were used to visualize variance between the young subject (left) and the aged subject (right). Figure 5 shows a summary of results for the matched younger and aged subjects in the study. Based on the analysis of the standard deviation of the heart rate values, it can be observed that a lower heart rate variability of $0.02 \mathrm{~s}$ was detected in the aged subject as compared with the younger subject (0.11). The 5-fold lower heart rate variability for the aged adult is indicative of greater arterial stiffness (Zhang, Zheng, Ma, \& Sun, 2011), which bears relevance to a person's cardiovascular performance.

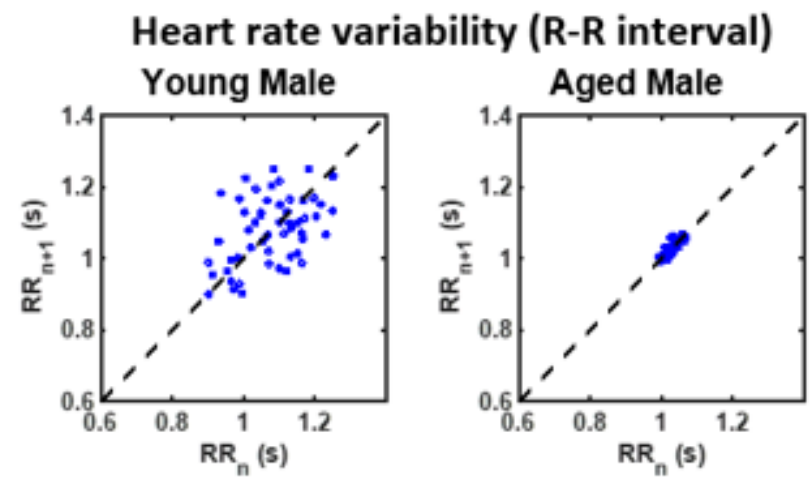

Figure 5. Heart rate patch sensor, and heart rate variability (Poincaré plots) results for the BMI and sex matched young (left) and aged (right) males of the study.

\subsection{Joint Flexibility Sensor}

Wearable sensors were also used to analyze the state of the subjects' joint flexibility. For this task, we utilized flexibility 
sensors, which are built of graphene films, whose resistance is sensitive to stretch. Figure 6 shows percentage change in the resistance of the flexibility sensor located in the hand joint of the BMI and gender-matched aged and younger individuals in our study. It can be observed that the maximum resistance changes of the young male upon bending his hand were over 7 times larger than the corresponding value for the aged male. These changes matched physical observations of the joint angle changes, which were significantly larger for the young male in comparison with the aged male, who suffered from arthritis.

\subsection{SmartPad sensors}

SmartPad raw measurements were used to assess metabolic rate data as well as environmental data related to comfort (temperature and relative humidity). Metabolic rate generally decreases with age in humans, and with all other factors held constant, is a primary driver of age related weight gain (Piers, Soares, McCormack, \& O’Dea, 1998). Figure 7A-B shows the metabolic rate assessment from the SmartPad sensor as described in the experimental sections. Figure 7C shows a summary of the metabolic rate values for the study subjects at sitting, biking and lying positions. It can be observed that the metabolic rates were significantly different between the young and aged subjects with similar BMI. Sudden drops in metabolic rate can lead to sudden weight gain in any individual, if caloric intake is kept constant (due to an increased positive overall caloric balance). Therefore, if these sudden changes in metabolic rate can be detected (in the frame of a few weeks/months), then the subject could potentially be immediately alerted and better able to manage their caloric needs via caloric tracking. On the contrary, if metabolic rate increases without a change in lifestyle (e.g., exercise), it may be a warning of increase of catabolic processes such as worsening of pulmonary obstructions (Agha \& El Wahsh, 2013; Hugli, Schutz, \& Fitting, 1996), cancer proliferation (Nguyen, Batterham, \& Edwards, 2016), and hormonal imbalances (Meunier et al., 2005; Mullur, Liu, \& Brent, 2014; Salomon et al., 1992). Figure 7D shows a custom-made reproduction from (McArdle, 2010) and serves to delineate the changes in metabolic rate due to age. In Figure 7D, the orange line represents metabolic rate for males as a function of age and the pink line represents the metabolic rate for females as a function of age. The differences of metabolic rate observed in Figure $7 \mathrm{C}$ are supported by the information provided in Figure 7D.

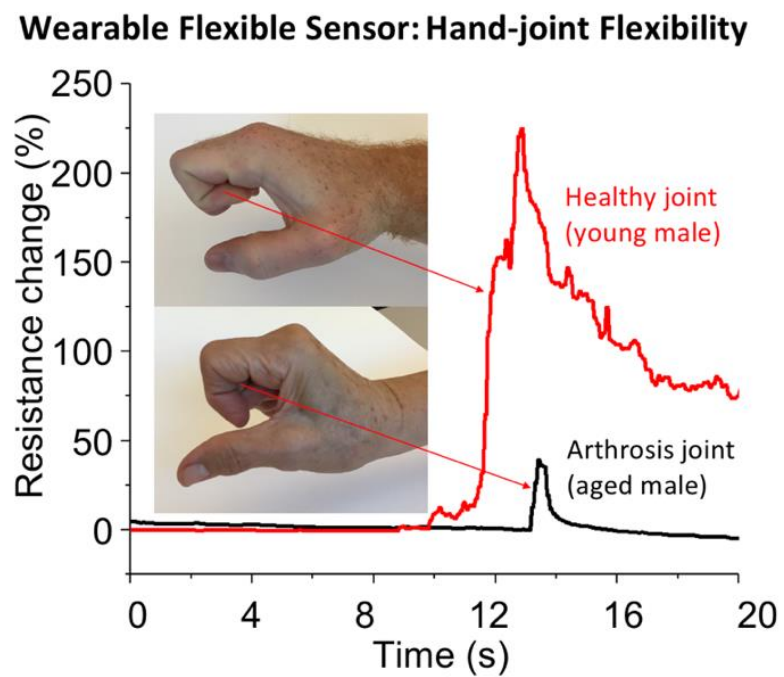

Figure 6. Wearable, flexible sensor to measure finger-joint flexibility showing a healthy joint from the young male, and an arthrosis-affected finger joint in an aged male.

\subsection{Smart Camera}

Figure 8 shows the results assessed with the smart camera obtained for the young and aged subjects of our study within the smart room. The results were simultaneously assessed with the aforementioned sensors, including the SmartPad. The subjects were video recorded during their biking activity. The smart camera software written in Python generated a realistic 3D model that encoded the various position of each subject's angle, knee, and hip joints among other joints. The positions were then mathematically transformed using algorithms to generate pitch (knee angle) vs. time data. These data sets were then analyzed using Fast Fourier Transforms (FFTs) to determine the frequency distribution pattern of the biking frequency (RPM) for each subject. Knowing the biking frequency and the bike resistance, the biking power was calculated and correlated to the SmartPad's measured metabolic rate to assess each subject's physical fitness level. It is worth noting that the physical fitness assessment of the study subjects could be exclusively performed by fusing the data from the smart sensors integrated in the SmartPad (chair) and the Smart Camera. The younger subject showed higher physical fitness than the older subject given that the younger subject was able to perform same amount of power with less energy expenditure and oxygen consumption rate. The lower weight adjusted metabolic rate to power production ratio should be interpreted as better physical fitness, since this ratio is a metric of the "energy investment of the person" (i.e. weight adjusted metabolic rate) to the "energy output of the person" (i.e. power output on the fixed bike). 


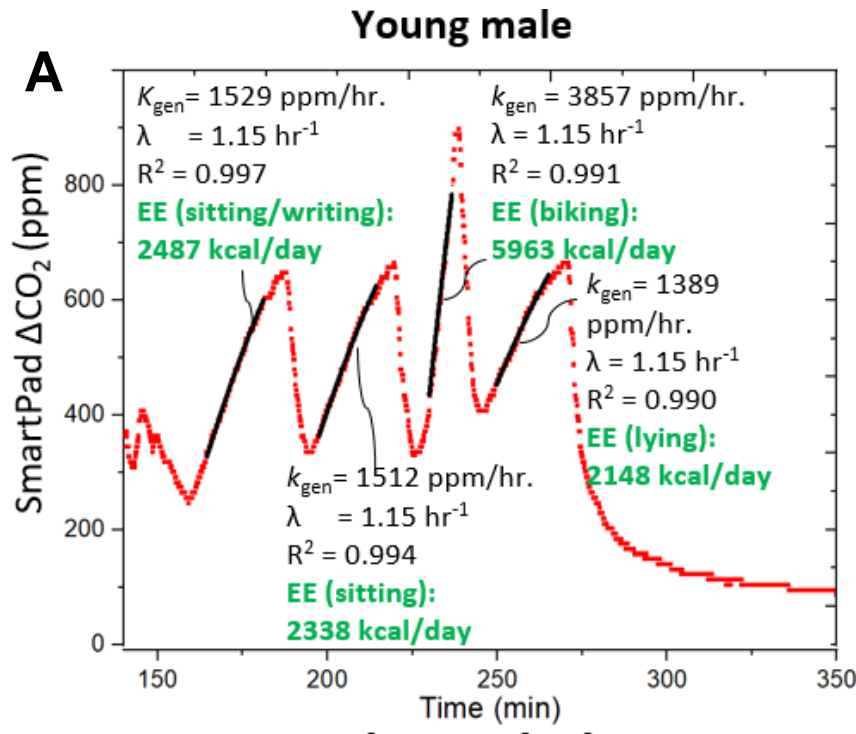

C SmartPad metabolic rate





D Long-term metabolic rate changes

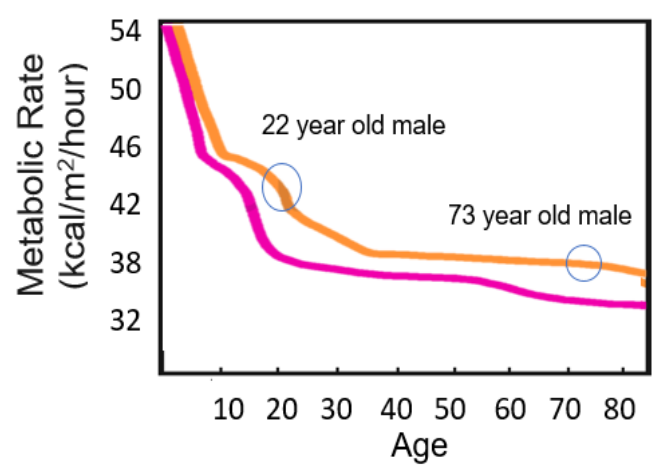

Figure 7. SmartPad sensor raw and fitted data for the young (A) and aged (B) subjects. The sawtooth appearance of the CO2 concentration over time is due to the nature of the system wherein an actuator system controls inlet/outlet fans leading into

the measurement environment. After each activity, the $\mathrm{CO} 2$ concentration within the room is reduced by turning the inlet/outlet fans ON, resulting in CO2 decay (Fig 7A-B). (C) Metabolic rate results corresponding to data in (A-B). (D) Data reconstructed from (McArdle, 2010): The curves represent population average metabolic rate for males (orange) and female (pink) as a function of age. 

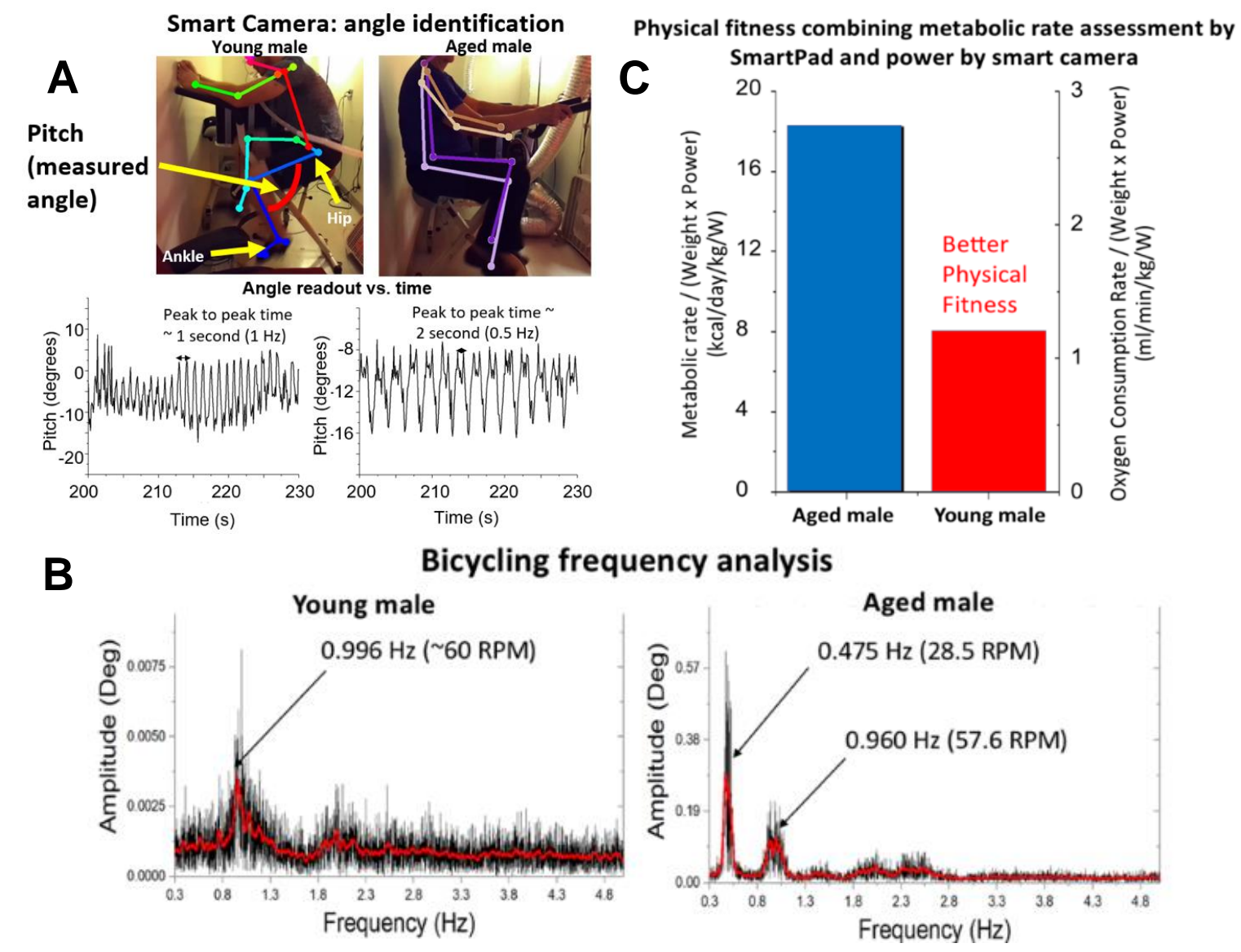

Fig. 8. (A) Smart camera images showing the joint angle identification, as well as the measurements of the pitch angle over time during biking for the two study subjects. (B) Fast Fourier Transform analysis of the data shown in (A), indicating the most frequent biking revolution-per-minute (RPM) for each subject. (C) Physical fitness assessment based on metabolic rate and oxygen consumption rate normalized by the subject's corresponding body weight and power production (from RPM and load measurement of bike).

\subsection{Smart bathroom sensors}

To evaluate the hydration state of the study subjects we used ion concentrations in urine as a proxy for osmolarity. It is well-known that the higher the ion concentrations in urine, the higher the osmolarity, and the higher the dehydration state of a subject (Armstrong et al., 1998). In this study, we evaluated the ammonia and potassium ion concentrations for urine samples obtained before and after the study challenge. Figure 9A-B shows the results, showing increases of $28 \%$ and $48 \%$ for ammonium concentration in the young male and aged subjects, respectively. In addition, the potassium concentration increased by $32 \%$ and $140 \%$ in the young and aged subjects, respectively. Furthermore, parallel tests performed with the same urine samples, quantifying specific gravity with a urine test strip, indicated an increase of 10 units for both subjects. However, the specific gravity increased to levels indicative of severe dehydration for the 73 year-old subject (Armstrong et al., 1998). Finally, the assessment of body weight in both subjects indicated a weight loss of $\sim 0.25 \mathrm{~kg}$ in the aged male, and $\sim 0.5 \mathrm{~kg}$ in the young male between the initial and final state of the challenge. Intuitively, it makes sense to expect this result (higher weight loss for young male) due to his higher metabolic rate and therefore weight loss from $\mathrm{CO}_{2}$ and vapor water exhalation. Specific gravity and weight changes were in coincidence with the ion concentration change results from the ion selective sensors of the smart toilet sensor, indicating the importance of these sensors to detect dehydration states. Dehydration was to be expected, as the subjects were not given any water during the experiment.

\subsection{In vitro methods}

\subsubsection{Telomere Length}

Telomeres are protective structures located at the ends of chromosomes and the length of telomeric DNA correlates with cellular aging. Over cell divisions, the telomeric DNA shortens (Rizvi, Raza, \& Mahdi, 2014; Shammas, 2011). As 
expected, the mean telomere length of the aged test subject was $6.9 \mathrm{~kb}$ which is significantly shorter than the $12.1 \mathrm{~kb}$ for the younger subject.

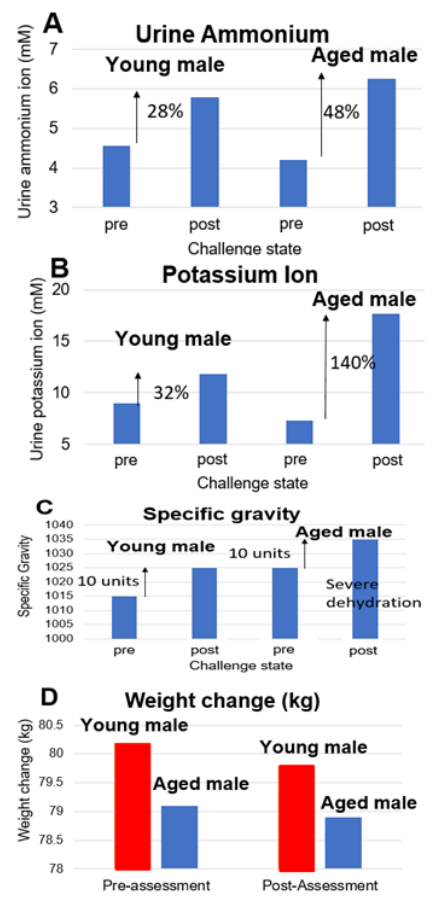

Figure 9. Smart toilet sensor, measurement of urine ammonium (A) and urine potassium (B) concentrations. Corresponding measurements of the urine specific gravity, and body weight for the young and aged male of the study.

\subsubsection{Atomic force microscopy (AFM) stiffness measurement of fruit fly heart tissue}

As shown in Figure 11, the stiffness of the heart of the aged and young flies was significantly different. Specifically, the heart tissues of the aged flies were significantly stiffer than that of the young flies: for each measured segment, the Young's modulus of the aged flies were at least three times higher than that of the young flies. Moreover, stiffness nonlinearity (i.e., stiffness changes due to measurement depth) of the aged fly heart tissues is much more significant. Particularly, the stiffness of the A1, A2, A3, and A4 of the aged flies increased by $44.7 \%, 11.2 \%, 14.1 \%$, and $89.3 \%$, respectively, when the indentation depth was double from $650 \mathrm{~nm}$. However, the corresponding stiffness increase of the young flies was less than $7 \%$ for all four measured segments. Note that the stiffness nonlinearity is a direct indicator of material homogeneity. These observations indicate that aging can lead to stiffening and increase the degree of heterogeneity of heart tissue, which are associated with many common heart diseases such as hypertrophic cardiomyopathy and ventricular arrhythmias.
The assessment of cellular stiffness was performed in fruit fly heart tissue as an illustrative example. Ongoing stiffness tests have been performed on human blood cells with similar outcomes (not shown in current publication) by the authors of this publication. In the future, we envision this test to be done by automated extraction of blood red cells by a simple finger prick sample collection, and automated cellular stiffness measurement.

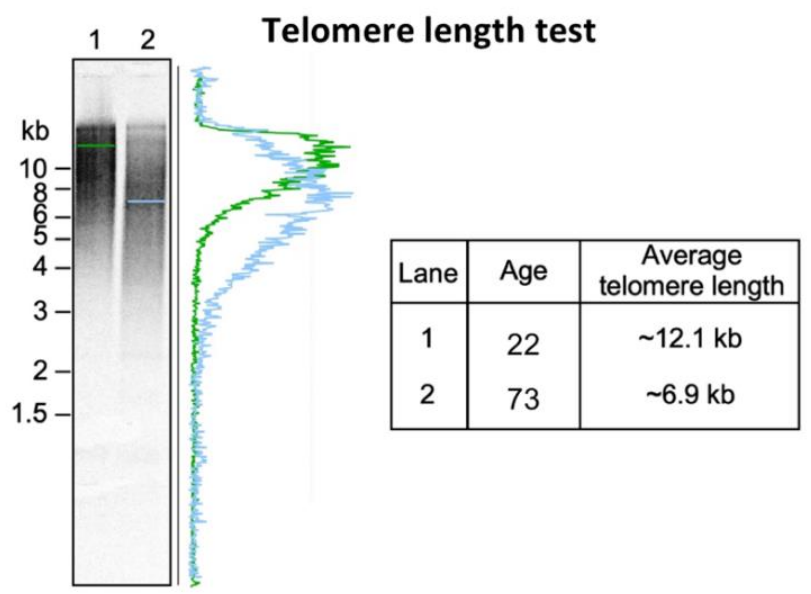

Figure 10. The mean telomere lengths of a younger (22 year- old) and aged (73 year-old) individual using telomere restriction fragment (TRF) length analysis. The TRF gel on the left shows the length distribution of restriction fragments of telomere DNA. Signal intensity traces of telomere fragments are shown on the right of the gel. Sizes of DNA markers are indicated on the left of the gel

\section{Conclusions}

The integration of multiple wearable and smart sensors into a single system enabled simultaneous assessment of physiological metrics. This capability allowed determination of key differences in the health status of individuals of different ages. Successful implementations of this type of system would enable assessment of baseline conditions over time to better understand overall wellness and to determine changes of health status or disease progression as well as understand real health problems among aged adults in connection with mobility, metabolic rate changes, dehydration, and activities of daily living. All of the sensing systems presented in this work require additional research in terms of validation and easy-to-use design but hold great promise for future applicability in assessing everyday functions of aged adults. A fully integrated, seamless system with all the sensors used is in progress, yet, the initial results presented herein show proof-of-concept for this integrated monitoring system of related physiological metrics. A key limitation of the study was the low sample size (only 2 subjects). Future works will strive to scale up the tested population and utilize parallel measurements of gold standard 
reference instruments for all collected metrics for comparison with the research level devices in this work.
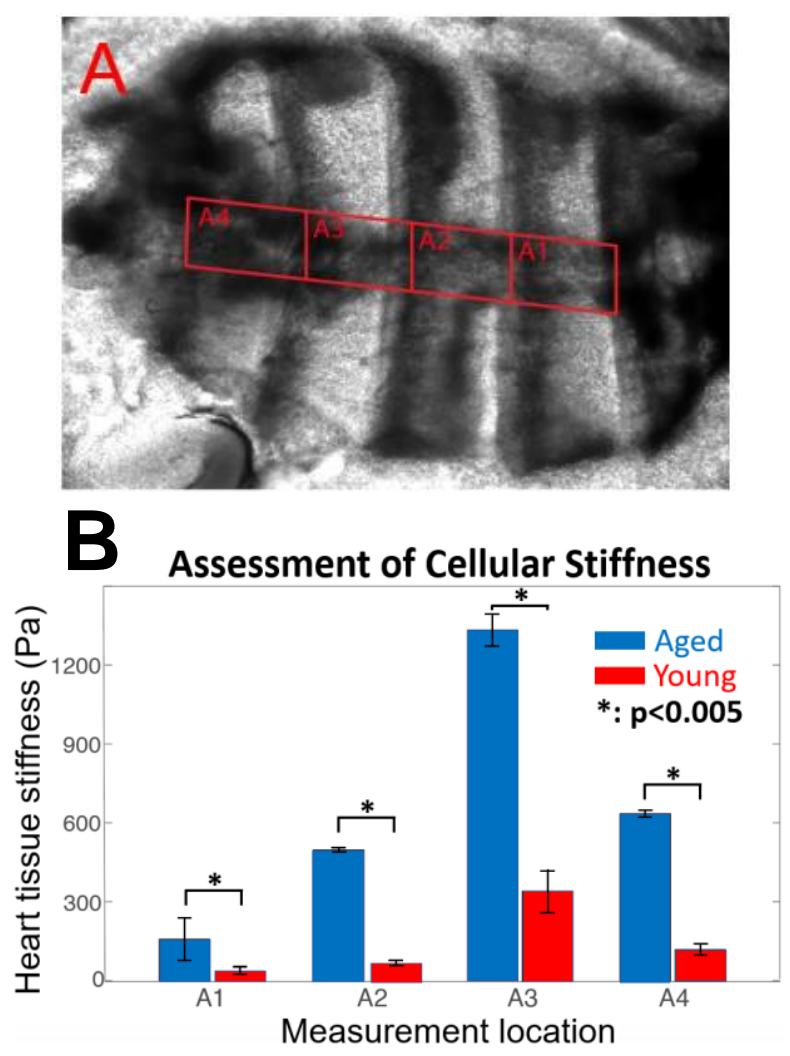

Figure 11. Cellular Stiffness Assessment. A: An example image of fruit fly heart tissue. Fig. 11B-C: A1, A2, A3 and $\mathrm{A} 4$ represents the measurement area of the fly heart Young's modulus different heart segments (i.e., A1, A2, A3, and A4) of the B) young flies and aged flies at the indentation depth of $1 \mu \mathrm{m} . \mathrm{n}>10$.

\section{ACKNOWLEDGEMENTS}

The Arizona State University Team would like to thank Kyle Squires, Paul Westerhoff, and the Ira A. Fulton School of Engineering for their support. The authors gratefully acknowledge funding support for this work by the National Science Foundation under award number CBET-1706994, ECCS-1841649, and the Iowa State University Nanovaccine Institute award number 2901738. Ivan Kucherenko thanks the United States Department of State Bureau of Educational and Cultural Affairs for the Fulbright fellowship (Fulbright Scholar Program, PS00267098). This work was also supported by an NIH Grant R01 GM094450. Erica Forzani acknowledges this work to A.J. and Sigimunda Palumbo Charitable Trust, and NIH-NIBIB (5R03EB027336) and Dr. NJ Tao, who encouraged her to pursue the integration of the teams' work, and supported the work performed in the Center for Bioelectronics and Biosensors at the Biodesign Institute, Arizona State University.

\section{REFERENCES}

Agha, M. A., \& El Wahsh, R. A. (2013). Basal metabolic rate in bronchial asthma and chronic obstructive pulmonary disease patients. Egyptian Journal of Chest Diseases and Tuberculosis, 62(1), 39-44. doi: https://doi.org/10.1016/j.ejcdt.2013.01.007

Armstrong, L. E., Soto, J. A., Hacker, F. T., Jr., Casa, D. J., Kavouras, S. A., \& Maresh, C. M. (1998). Urinary indices during dehydration, exercise, and rehydration. International Journal of Sport Nutrition, 8(4), 345-355. doi:10.1123/ijsn.8.4.345

Barns, S., Balanant, M. A., Sauret, E., Flower, R., Saha, S., \& Gu, Y. (2017). Investigation of red blood cell mechanical properties using AFM indentation and coarse-grained particle method. Biomedical engineering online, 16(1), 140-140. doi:10.1186/s12938-017-0429-5

Benetos, A., Gardner, J. P., Zureik, M., Labat, C., Xiaobin, L., Adamopoulos, C., . . . Aviv, A. (2004). Short telomeres are associated with increased carotid atherosclerosis in hypertensive subjects. Hypertension, 43(2), 182-185. doi:10.1161/01.HYP.0000113081.42868.f4

Bennett, J., Thomas, V., \& Riegel, B. (2004). Unrecognized Chronic Dehydration in Older Adults: Examining Prevalence Rate and Risk Factors. Journal of gerontological nursing, 30, 22-28; quiz 52. doi:10.3928/0098-9134-20041101-09

Binette, J., \& Vasold, K. (2018). 2018 Home and Community Preferences: A National Survey of Adults Age 18-Plus. doi: https://doi.org/10.26419/res.00231.001

Boulgarides, L. K., McGinty, S. M., Willet, J. A., \& Barnes, C. W. (2003). Use of clinical and impairment-based tests to predict falls by community-dwelling older adults. Physical Therapy, 83(4), 328-339.

Brouilette, S., Singh, R. K., Thompson, J. R., Goodall, A. H., \& Samani, N. J. (2003). White cell telomere length and risk of premature myocardial infarction. Arterioscler Thromb Vasc Biol, 23(5), 842-846. doi:10.1161/01.atv.0000067426.96344.32

Chan, E., Hu, Y., Johnson, P., Suo, Z., \& Stafford, C. (2012). Spherical indentation testing of poroelastic relaxations in thin hydrogel layers. Soft Matter, 8, 1492-1498. doi:10.1039/C1SM06514A

Charlton, P. H., Bonnici T Fau - Tarassenko, L., Tarassenko L Fau - Alastruey, J., Alastruey J Fau - Clifton, D. A., Clifton Da Fau - Beale, R., Beale R Fau - Watkinson, P. J., \& Watkinson, P. J. (2017). Extraction of respiratory signals from the electrocardiogram and photoplethysmogram: technical and physiological determinants. Physioloigical Measurement(1361-6579 (Electronic)).

Chu, M., Nguyen, T., Pandey, V., Zhou, Y., Pham, H. N., Bar-Yoseph, R., . . Khine, M. (2019). Respiration rate and volume measurements using wearable strain sensors. 
NPJ Digital Medicine, 2, 8. doi:10.1038/s41746-0190083-3

Daubney, M. E., \& Culham, E. G. (1999). Lower-extremity muscle force and balance performance in adults aged 65 years and older. Physical Therapy, 79, 1177-1185.

Dimitriadis, E. K., Horkay, F., Maresca, J., Kachar, B., \& Chadwick, R. S. (2002). Determination of elastic moduli of thin layers of soft material using the atomic force microscope. Biophysical journal, 82(5), 2798-2810. doi:10.1016/S0006-3495(02)75620-8

Doheny, E. P., McGrath, D., Greene, B. R., Walsh, L., McKeown, D., Cunningham, C., . . Caulfield, B. (2012). Displacement of centre of mass during quiet standing assessed using accelerometry in older fallers and non-fallers. Conf Proc IEEE Eng Med Biol Soc, 2012, 3300-3303. doi:10.1109/embc.2012.6346670

Frames, C. W., Soangra, R., Lockhart, T. E., Lach, J., Ha, D. S., Roberto, K. A., \& Lieberman, A. (2018). Dynamical Properties of Postural Control in Obese CommunityDwelling Older Adults. Sensors, 18(6), 1692. Retrieved from https://www.mdpi.com/1424-8220/18/6/1692

Gago, M. F., Fernandes, V., Ferreira, J., Silva, H., Rocha, L., Bicho, E., \& Sousa, N. (2014). Postural stability analysis with inertial measurement units in Alzheimer's disease. Dementia and geriatric cognitive disorders extra, 4(1), 22-30. doi:10.1159/000357472

Gohring, J., Fulcher N Fau - Jacak, J., Jacak J Fau - Riha, K., \& Riha, K. (2014). TeloTool: a new tool for telomere length measurement from terminal restriction fragment analysis with improved probe intensity correction. Nucleic Acids Res.

(1362-4962 (Electronic)).

Hugli, O., Schutz, Y., \& Fitting, J. W. (1996). The daily energy expenditure in stable chronic obstructive pulmonary disease. American Journal of Respiratory and Critical Care Medicine, 153(1), 294-300. doi:10.1164/ajrccm.153.1.8542132

Kanazawa, A., Black, M. J., Jacobs, D. W., \& Malik, J. (2018, 18-23 June 2018). End-to-End Recovery of Human Shape and Pose. Paper presented at the 2018 IEEE/CVF Conference on Computer Vision and Pattern Recognition.

Kim, J., Chou, E.-F., Le, J., Wong, S., Chu, M., \& Khine, M. (2019). Soft Wearable Pressure Sensors for Beat-to-Beat Blood Pressure Monitoring. Advanced Healthcare Materials, $\quad 8(13), \quad 1900109$. doi:10.1002/adhm.201900109

Lavizzo-Mourey, R. J. (1987). Dehydration in the elderly: a short review. Journal of the National Medical Association, 79(10), 1033-1038. Retrieved from https://www.ncbi.nlm.nih.gov/pubmed/3316670

https://www.ncbi.nlm.nih.gov/pmc/articles/PMC2625510/

Lockhart, T. E., Soangra, R., \& Frames, C. (2014). Fall risks assessment among community dwelling elderly using wearable wireless sensors (Vol. 9091): SPIE.
Loper, M., Mahmood, N., Romero, J., Pons-Moll, G., \& Black, M. J. (2015). SMPL: a skinned multi-person linear model. ACM Trans. Graph., 34(6), 1-16. doi:10.1145/2816795.2818013

McArdle, W. D. K., F. I.; Katch, V. L. (2010). Exercise physiology: nutrition, energy, and human performance.: Lippincott Williams \& Wilkins.

Melzer, I., Benjuya, N., \& Kaplanski, J. (2004). Postural stability in the elderly: a comparison between fallers and non-fallers. Age Ageing, 33(6), 602-607. doi:10.1093/ageing/afh 218

Mender, I., \& Shay, J. W. (2015). Telomere Restriction Fragment (TRF) Analysis. Bio-protocol, 5(22), e1658. doi:10.21769/bioprotoc. 1658

Meunier, N., Beattie, J. H., Ciarapica, D., O'Connor, J. M., Andriollo-Sanchez, M., Taras, A., . . Polito, A. (2005). Basal metabolic rate and thyroid hormones of latemiddle-aged and older human subjects: the ZENITH study. European Journal of Clinical Nutrition, 59(2), S53-S57. doi:10.1038/sj.ejen.1602299

Mollaeian, K., Liu, Y., Bi, S., Wang, Y., Ren, J., \& Lu, M. (2018). Nonlinear Cellular Mechanical Behavior Adaptation to Substrate Mechanics Identified by Atomic Force Microscope. Int J Mol Sci, 19(11). doi:10.3390/ijms 19113461

Mora, S. J., Bridgeman, D., Xian, X., Tao, N., \& Forzani, E. (2020). Validation of Resting Energy Expenditure (REE) Measurement of new Breezing Pro device through Douglas Bag Method. Global Journal of Obesity, Diabetes and Metabolic Syndrome, 7(1).

Mullur, R., Liu, Y.-Y., \& Brent, G. A. (2014). Thyroid hormone regulation of metabolism. Physiological reviews, $\quad 94(2), \quad 355-382$. doi:10.1152/physrev.00030.2013

Musich, S., Wang, S. S., Ruiz, J., Hawkins, K., \& Wicker, E. (2018). The impact of mobility limitations on health outcomes among older adults. Geriatric Nursing, 39, 162-169.

Nguyen, T. Y. V., Batterham, M. J., \& Edwards, C. (2016). Comparison of Resting Energy Expenditure Between Cancer Subjects and Healthy Controls: A MetaAnalysis. Nutrition and Cancer, 68(3), 374-387. doi:10.1080/01635581.2016.1153667

Older People Projected to Outnumber Children for First Time in U.S. History. (2018).

Oren, S., Ceylan, H., Schnable, P. S., \& Dong, L. (2017). High-Resolution Patterning and Transferring of Graphene-Based Nanomaterials onto Tape toward Rollto-Roll Production of Tape-Based Wearable Sensors. Advanced Materials Technologies, 2(12), 1700223. doi:10.1002/admt.201700223

Panossian, L. A., Porter, V. R., Valenzuela, H. F., Zhu, X., Reback, E., Masterman, D., . . Effros, R. B. (2003). Telomere shortening in $\mathrm{T}$ cells correlates with Alzheimer's disease status. Neurobiol Aging, 24(1), 7784. doi:10.1016/s0197-4580(02)00043-x 
Perdue, P. W., Watts Dd Fau - Kaufmann, C. R., Kaufmann Cr Fau - Trask, A. L., \& Trask, A. L. (1998). Differences in mortality between elderly and younger adult trauma patients: geriatric status increases risk of delayed death. Journal of Trauma(0022-5282 (Print)).

Picetti, D., Foster, S., Pangle, A. K., Schrader, A., George, M., Wei, J. Y., \& Azhar, G. (2017). Hydration health literacy in the elderly. Nutrition and healthy aging, 4(3), 227-237. doi:10.3233/NHA-170026

Piers, L. S., Soares, M. J., McCormack, L. M., \& O’Dea, K. (1998). Is there evidence for an age-related reduction in metabolic rate? Journal of Applied Physiology, 85(6), 2196-2204. doi:10.1152/jappl.1998.85.6.2196

Popkin, B. M., D'Anci Ke Fau - Rosenberg, I. H., \& Rosenberg, I. H. (2010). Water, hydration, and health. Nutrition Reviews(1753-4887 (Electronic)).

Riebl, S. K., \& Davy, B. M. (2013). The Hydration Equation: Update on Water Balance and Cognitive Performance. ACSM's health \& fitness journal, 17(6), 21-28. doi:10.1249/FIT.0b013e3182a9570f

Rizvi, S., Raza, S. T., \& Mahdi, F. (2014). Telomere length variations in aging and age-related diseases. Curr Aging Sci, $\quad 7(3), \quad 161-167$. doi:10.2174/1874609808666150122153151

Ruiz, I., Sprowls, M., Deng, Y., Kulick, D., Destaillats, H., \& Forzani, E. S. (2018). Assessing metabolic rate and indoor air quality with passive environmental sensors. Journal of Breath Research, 12(3), 036012. doi:10.1088/1752-7163/aaaec9

Salomon, F., Cuneo Rc Fau - Hesp, R., Hesp R Fau - Morris, J. F., Morris Jf Fau - Poston, L., Poston L Fau - Sonksen, P. H., \& Sonksen, P. H. (1992). Basal metabolic rate in adults with growth hormone deficiency and in patients with acromegaly: relationship with lean body mass, plasma insulin level and leucocyte sodium pump activity. Clinical Science(0143-5221 (Print)).

Shammas, M. A. (2011). Telomeres, lifestyle, cancer, and aging. Current opinion in clinical nutrition and metabolic care, 14(1), 28-34. doi:10.1097/MCO.0b013e32834121b1

Shumway-Cook, A., Brauer, S., \& Woollacott, M. (2000). Predicting the probability for falls in communitydwelling older adults using the Timed Up \& Go Test. Physical Therapy, 80(9), 896-903.

Woods, A. L., Garvican-Lewis, L. A., Lundy, B., Rice, A. J., \& Thompson, K. G. (2017). New approaches to determine fatigue in elite athletes during intensified training: Resting metabolic rate and pacing profile. $P L O S$ One, 12(3), e0173807. doi:10.1371/journal.pone.0173807

Zhang, Y. L., Zheng, Y. Y., Ma, Z. C., \& Sun, Y. N. (2011). Radial pulse transit time is an index of arterial stiffness. Hypertens Res, 34(7), 884-887. doi:10.1038/hr.2011.41 